\title{
Undifferentiated small round cell sarcoma in a young male: a case report
}

\author{
Cora A. Ricker, ${ }^{1,6}$ Noah E. Berlow, ${ }^{1,6}$ Kenneth A. Crawford, ${ }^{1}$ \\ Todd Georgopapadakos, ${ }^{1}$ Audrey N. Huelskamp, ${ }^{1}$ Andrew D. Woods, ${ }^{1}$ \\ Eugen Dhimolea, ${ }^{2}$ Shakti H. Ramkissoon, ${ }^{3}$ Sheri L. Spunt, ${ }^{4}$ Erin R. Rudzinski, ${ }^{5}$ \\ and Charles Keller ${ }^{1}$
}

\begin{abstract}
${ }^{1}$ Children's Cancer Therapy Development Institute, Beaverton, Oregon 97005, USA; ${ }^{2}$ Dana-Farber Cancer Institute, Boston, Massachusetts 02215, USA; ${ }^{3}$ Foundation Medicine Inc., Cambridge, Massachusetts 02141, USA; ${ }^{4}$ Department of Pediatrics, Stanford University School of Medicine, Palo Alto, California 94304, USA; ${ }^{5}$ Seattle Children's Hospital, Seattle, Washington 98105, USA
\end{abstract}

\begin{abstract}
CIC-rearranged sarcomas (CRSs) have recently been characterized as a distinct sarcoma subgroup with a less favorable prognosis compared to other small round cell sarcomas. CRSs share morphologic features with Ewing's sarcoma and prior to 2013 were grouped under undifferentiated sarcomas with round cell phenotype by the WHO classification. In this report, whole-genome sequencing and RNA sequencing were performed for an adolescent male patient with CRS who was diagnosed with undifferentiated pleomorphic sarcoma (UPS) by three contemporary institutions. Somatic mutation analysis identified mutations in IQGAP1, CCNC, and ATXN1L in pre- and post-treatment tissue samples, as well as a CIC-DUX4 fusion that was confirmed by qPCR and DUX4 immunohistochemistry. Of particular interest was the overexpression of the translation factor eEF1A1, which has oncogenic properties and has recently been identified as a target of the investigational agent plitidepsin. This case may provide a valuable waypoint in the understanding and classification of CRSs and may provide a rationale for targeting eEF1A1 in similar soft tissue sarcoma cases.
\end{abstract}

[Supplemental material is available for this article.]

Corresponding author: charles@cc-tdi.org

(c) 2020 Ricker et al. This article is distributed under the terms of the Creative Commons Attribution-NonCommercial License, which permits reuse and redistribution, except for commercial purposes, provided that the original author and source are credited.

Ontology term: Ewing's sarcoma

Published by Cold Spring Harbor Laboratory Press

doi:10.1101/mcs.a004812

\section{INTRODUCTION}

Because of the rarity of each non-rhabdomyosarcoma soft tissue sarcoma (NRSTS) subtype, clinical investigation of NRSTS aggregates children with many diagnoses into the same clinical trial. Synovial sarcoma, malignant peripheral nerve sheath tumor (MPNST), fibrosarcoma, undifferentiated pleomorphic sarcoma (UPS), and CIC-rearranged sarcoma (CRS) are just a few of more than 50 NRSTSs grouped together for pediatric and adult clinical trials. Survival rates for NRSTSs can be poor when tumors are high-grade, large $(>5 \mathrm{~cm})$, difficult to resect in their entirety, and/or metastatic (Spunt et al. 2008; Ferrari et al. 2011).

Until 2013, CRS was classified as an undifferentiated sarcoma by the World Health Organization (WHO) (Doyle 2014). Because CRSs share similar morphological features to Ewing's sarcoma, these NRSTSs are often considered "Ewing's sarcoma-like" and historically may have followed similar treatment protocols (Doyle 2014). Morphologically, CRSs are characterized by their medium round or ovoid cells and lack of differentiation, with rare cases

\footnotetext{
${ }^{6}$ These authors contributed equally to this work.
} 
having an epithelioid appearance and mild nuclear pleomorphism (Italiano et al. 2012; Antonescu et al. 2017). However, further clinical and molecular evidence has distinguished CRSs as a distinct new subgroup because of their clinicopathologic and molecular features (Antonescu et al. 2017).

Here, we present the case of a young adolescent male patient initially diagnosed with undifferentiated epithelioid and pleomorphic sarcoma posthumously confirmed to be a high-grade CRS. Prior to the identification of the CIC-DUX4 rearrangement, the patient was treated as an UPS, which has varying clinical treatment approaches that are minimally effective. Broadly, the treatment for CRS is poorly defined. Previous studies suggest that patients with the CIC-DUX4 gene fusion from either a $\mathrm{t}(4: 19)$ or $\mathrm{t}(10: 19)$ translocation tend to have an aggressive clinical course with metastatic relapse in 61\% (11/18) of cases at follow-up (Italiano et al. 2012). Other CRS translocations include CIC-FOXO4 or CIC-NUTM1 fusions, but these alternative fusions account for only 5\% of CRSs (Watson et al. 2018). Although these new findings suggest that the $\mathrm{CIC}$ translocation is a driver of tumor growth and metastasis in CRS, the translocation is currently not targetable. To further advance translational insights for CRS, we have investigated the whole-genome and RNA sequencing of a young adolescent with CRS.

\section{RESULTS}

\section{Clinical Presentation}

An 11-yr-old male first presented with a soft tissue mass in the proximal left lower leg, which was initially diagnosed as a ganglion cyst. Because the mass continued to grow, a second opinion was sought $\sim 3$ mo later. After magnetic resonance imaging (MRI) confirmed the presence of a solid tumor (Fig. 1; Supplemental Fig. 1), he underwent excisional biopsy. Histologic examination of the mass demonstrated epithelioid-to-spindled cells distributed in sheets with alternating cellular and hypocellular fibrotic areas (Fig. 2A-C). Large scattered cells with abundant eosinophilic cytoplasm were present, whereas other areas demonstrated sheets of smaller tumor cells with a more primitive appearance within a looser, myxoid
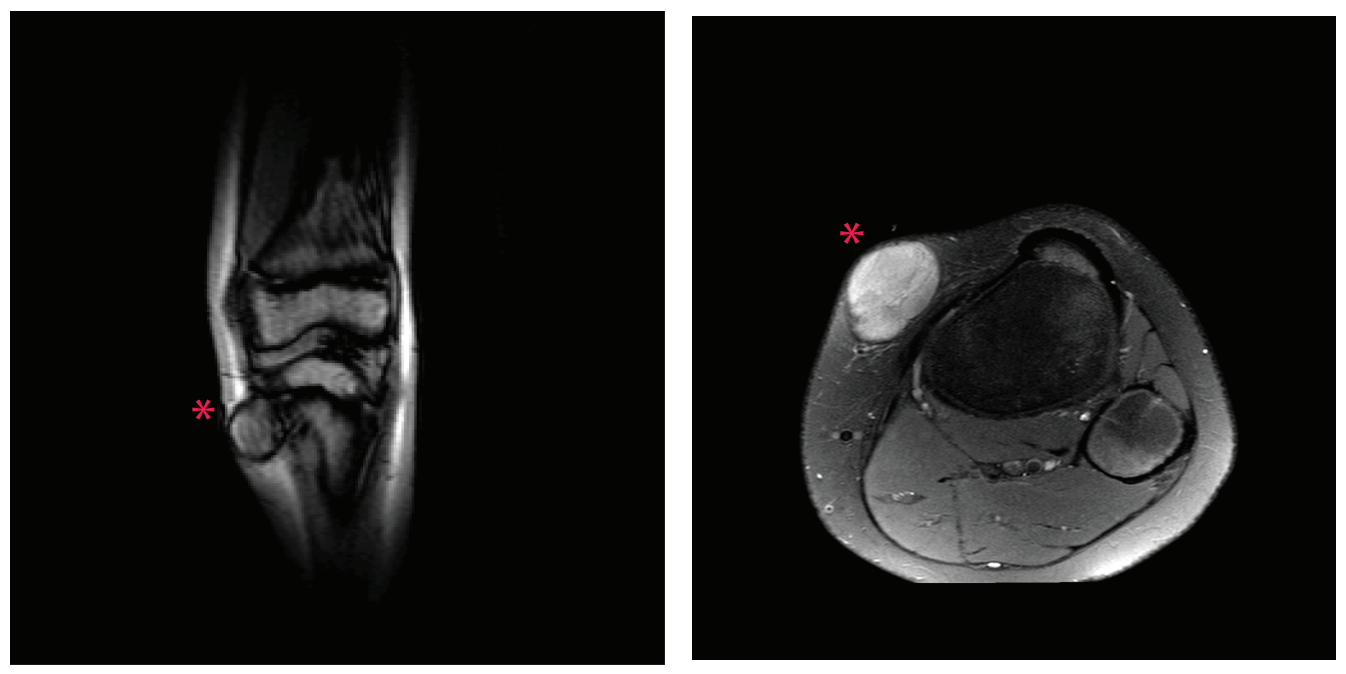

Figure 1. MRI scans of the left knee. A well-defined elliptical lesion (red asterisk) in the subcutaneous fat anteromedial to the proximal tibia (right) was found with the following multiplanar sequences: survey (left) and lateral tibia axial proton density with fat saturation (right). 
A

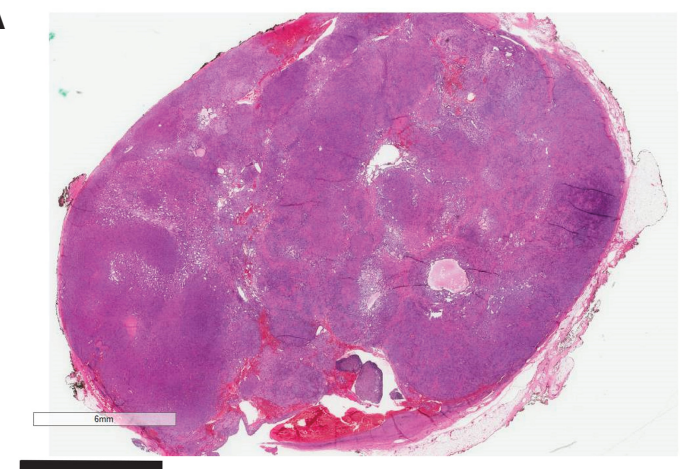

C

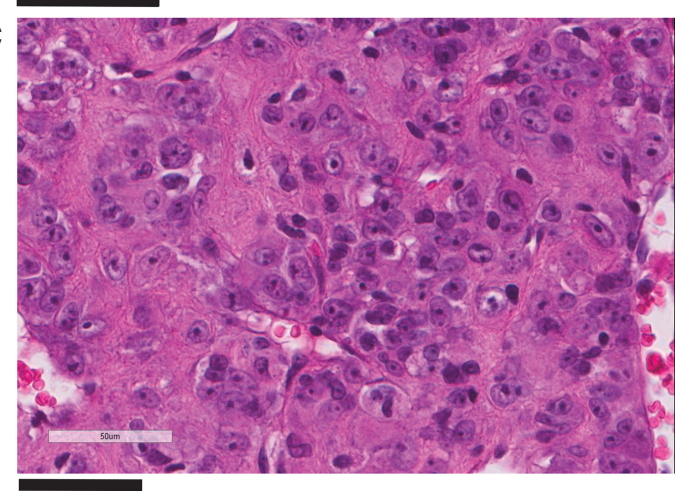

B

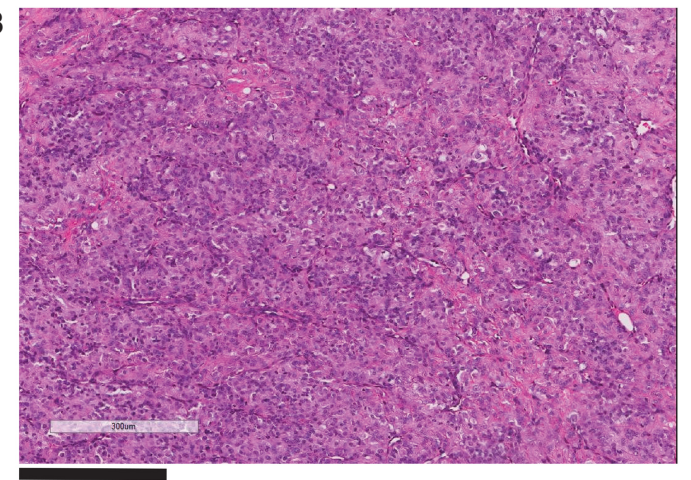

D

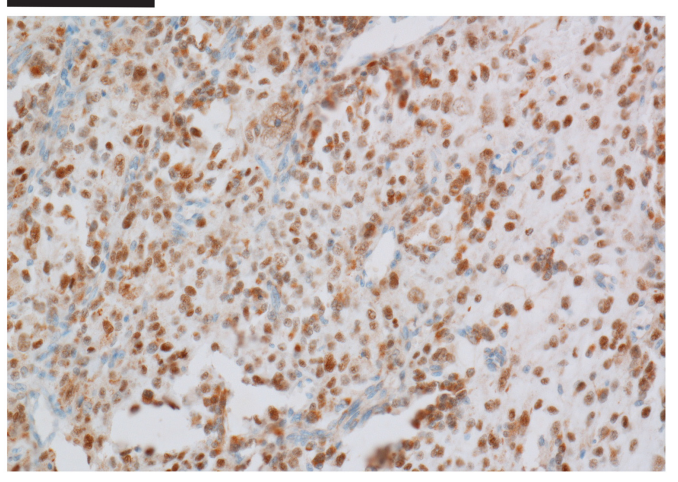

Figure 2. Morphological diagnostic immunohistochemistry for $C / C$-rearranged sarcoma. $(A-C)$ Hematoxylin and eosin (H\&E) staining of the patient's tumor section revealed a pleomorphic high-grade $\mathrm{ClC}$-rearranged sarcoma. Scale bars, $6 \mathrm{~mm}(A), 300 \mu \mathrm{M}(B)$, and $50 \mu \mathrm{M}(C)$. (D) DUX4 immunohistochemistry staining.

matrix. The tumor nuclei were polygonal with round to oval nuclei, vacuolated chromatin, and prominent nucleoli. Immunostaining revealed cells that were positive for CD99 and vimentin and negative for SMA, desmin S100, CD34, MyoD1, AE1/AE3, and CD31. Thus, no evidence was found to support any line of differentiation, including leiomyosarcoma, rhabdomyosarcoma, epithelioid sarcoma, perivascular epithelial cell tumor, nor myoepithelial neoplasm. The tumor was ultimately classified as an undifferentiated epithelioid and pleomorphic sarcoma, and the patient underwent treatment for a UPS.

Five weeks after the initial surgery, the patient underwent primary re-excision that achieved complete margins. A metastatic workup including chest computed tomography (CT) and bone scan was negative. The patient was subsequently enrolled in Children's Oncology Group study ARST0332, in which he was observed without adjuvant therapy. Tumor surveillance included an MRI of the primary tumor region every 3 mo and a chest $X$ ray every 6 mo. At month 11 after diagnosis, the patient developed left shoulder pain and a persistent cough and was diagnosed with pneumonia. Subsequent imaging due to symptom persistence demonstrated bilateral pulmonary and pleural metastases with a right pleural effusion. After short-term pleural fluid drainage with a chest tube, the patient received ifosfamide/doxorubicin chemotherapy for a total of six cycles (cumulative $54 \mathrm{~g} / \mathrm{m}^{2}$ ifosfamide, 375 $\mathrm{mg} / \mathrm{m}^{2}$ doxorubicin). A minor tumor response was documented after three cycles of chemotherapy, and right thoracotomy with complete resection of the right-sided lung nodules was performed. Pathology showed an extensive treatment effect with a $<5 \%$ viable tumor.

Tumor tissue from the right thoracotomy was sent for next-generation panel sequencing using the FoundationOne Heme test, which identifies genomic alterations in 405 genes (median exon coverage of $\sim 500 \times)$. Alterations were found in CHD2, FOXO1, HDAC7, RPTOR, 
COLD SPRING HARBOR Molecular Case Studies
Translation factor eEF1A1 overexpression in a ClC-rearranged sarcoma case
TSC2, and WDR90, all with unknown significance (Supplemental Table 1). Thus, no actionable genomic alterations were found. Tissue samples from the first excision of the right lung metastatic tumor were also sent to Human Longevity Inc. (HLI) for whole-genome sequencing (WGS) (113x coverage) and RNA sequencing together revealed no cancer-relevant somatic alterations or copy-number variations (CNVs). Overall WGS mutation rate was $<1$ mutation per megabase (MB). Of note, because of the low tumor purity observed in the samples, the sensitivity to capture low allele fractions variants was reported to be suboptimal; thus, some low-frequency alterations may not be called. In addition, copy-number alterations could not be assessed because of the tumor purity. Quantitative polymerase chain reaction (qPCR) studies at $\mathrm{HLI}$ raised the question of a CIC-DUX4 fusion and the suggestion of FISH studies, which is discussed in a later section below.

After completion of the remaining chemotherapy, a left thoracotomy and resection of the remaining lung nodules were performed. Pathology reported microscopic residual disease at the margins of two resected lung nodules. Fresh frozen tumor samples were sent from the left thoracotomy to $H L I$ for WGS $(127 \times$ coverage), whole-exome sequencing (WES, 574 $\times$ coverage), RNA sequencing ( $80 \mathrm{M}$ reads), Oncomine panel, and qPCR. Again, no cancer-relevant single-nucleotide variants (SNVs), indels, or CNVs were reported. However, the samples each had low tumor purities based on pathological assessments of the second sample tested and computational assessments performed on all three samples. An orthogonal sequencing method using Oncomine Comprehensive Assay (OCAv2) with Ion Torrent technology (ThermoFisher Scientific) was also performed and did not detect any SNVs, indels, or fusions. The samples were found to have a low mutational burden with two mutations per megabase (Mb). Evidence of a potential CIC-DUX4 fusion, described as translocation $t(4 ; 19)(q 35 ; q 13.1)$, was identified in the FFPE sample and the fresh frozen sample by WGS (four reads/sample) and confirmed by qPCR in the fresh frozen RNA sample: Two out of the three sets of primers designed to span the predicted fusion junctions produced a positive result amplifying a fusion junction in CIC-DUX4. Blood was also sent as a normal control for WGS with $31 \times$ and WES with $227 \times$. No pathogenic variants associated with inherited cancer risks and no variants associated with adverse drug reaction or drug metabolism were reported. HLI reported relative quantities of PD-1, CTLA4, and NYESO-1 from qPCR and provided the patient's Human Leukocyte Antigen (HLA) typing (Tables 1 and 2).

\begin{tabular}{lcc}
\hline \multicolumn{2}{l}{ Table 1. Human Longevity expression results } & \\
\hline Gene of interest & Expression level (TPM) & Percentile in TCGA: SARC (\%) \\
\hline PD-1 & 26.9 & 99 \\
PD-L1 & 3.1 & 84 \\
PD-L2 & 10.2 & 87 \\
CTLA4 & 9.5 & 100 \\
LAG3 & 8.2 & 79 \\
B7-H3 & 242.4 & 70 \\
NY-ESO-1 & 0.3 & 83 \\
CD3 & 1.8 & 78 \\
HER2 & 40.3 & 90 \\
INI-1 & 570.7 & 100 \\
GM2/GD2 synthase & 0.5 & 8 \\
\hline
\end{tabular}

(TPM) Transcripts per million, (TCGA) The Cancer Genome Atlas, (SARC) Sarcoma Alliance for Research through Collaboration. 
COLD SPRING HARBOR Molecular Case Studies
Translation factor eEF1A1 overexpression in a CIC-rearranged sarcoma case

Treatment with pazopanib was initiated two months later upon adequate postoperative recovery. A CT scan two months after initiation of pazopanib revealed tumor regrowth in the patient's chest wall/diaphragm and superior mediastinum leading to discontinuation of pazopanib and initiation of pembrolizumab, with palliative radiation therapy (RT) to the right chest wall and right superior mediastinal tumors. The rationale for this therapeutic approach was a report of objective responses to pembrolizumab in adults with UPS (Tawbi et al. 2016), along with early evidence of abscopal effects from combining radiotherapy with PD-1 antibody. Further, immunotherapy was given off-trial because previous studies have shown a rich immune infiltrate in adults with UPS; however, such an infiltrate was not seen in this case.

Two months after initiation of pembrolizumab, following radiation and two doses of pembrolizumab, imaging showed a mixed response with shrinkage of the right chest tumor (within the radiotherapy field) and emergence of a new right lower lobe pulmonary metastasis. The patient underwent resection of the mediastinal, right lower lobe, and right chest wall tumors with en bloc resection of ribs $5-8$ and the anterior diaphragm with bioprosthetic mesh reconstruction. Pathology showed complete but close margins, and pembrolizumab was resumed. Two months later, a CT scan showed a new soft tissue mass lateral to the liver near the surgical bed. Pembrolizumab was discontinued and the tumor was treated with 40 Gy of stereotactic body radiation therapy in six fractions. Over the subsequent 7 mo, slowly progressive lung metastases developed, and the patient subsequently was enrolled on a phase I trial of the B7H3-targeted antibody MGA-271. He developed multiple brain and leptomeningeal metastases prompting discontinuation of MGA-271 and initiation of steroids and whole-brain radiotherapy. Imaging confirmed systemic disease progression in the lungs, chest wall, and intrathoracic lymph nodes. Despite improvement in neurologic function, death occurred as a result of respiratory failure related to intrathoracic disease progression.

After the patient's death, autopsy noted extensive UPS metastatic disease involving the heart, diaphragm, right chest wall, small bowel, both kidneys, liver, spleen, and pulmonary hilar lymph nodes. Posthumously, a CIC-rearranged sarcoma diagnosis was later rendered based on the presence of diffuse DUX4 nuclear staining by immunohistochemistry (Fig. 2D), which confirmed the CIC-DUX4 rearrangement identified by NGS and qPCR.

\section{Genomic Analyses}

To identify possible genomic mutations and investigate the relationship between pre- and post-treatment tumors, WGS was performed on the tumor samples obtained from the 
COLD SPRING HARBOR Molecular Case Studies
Translation factor eEF1A1 overexpression in a CIC-rearranged sarcoma case patient. We analyzed tumor and matched normal genome sequencing data for the presence of somatic point mutation, somatic functional and structural mutations, potential germline mutations, polynucleotide insertions/deletions, and gene CNVs. After filtering the list against germline sequence variants, 75 somatic mutations and 16 potential germline mutations were identified. Fourteen pretreatment mutations of interest were identified, and 17 post-treatment genes of interest were identified, with 11 mutations in common (Table 3; Figs. 3 and 4). IQGAP1, CCNC, and ATXN1L were identified as genes bearing high- or medium-impact mutations in the pretreatment sample that were not found in the matched normal genome. RNA sequencing revealed significant up-regulation of VGF, AP1M2, and ETV4 from pretreatment to post-treatment. ETV4 is often up-regulated in CRS and is a frequent diagnostic immunohistochemistry (IHC) tool (Hung et al. 2016). Pretreatment and post-treatment were both 91 nonsynonymous mutations across all exonic regions (Table 4).

Gene fusions were also identified using RNA sequencing from the pre- and post-treatment samples. Equivocal evidence was found for the EEF1A1-RPL32 fusion gene in the post-treatment, but not in the pretreatment tumor sample (Figs. 3 and 4; Supplemental Table 2). A CIC-DUX4 fusion could not be called by the standard calling parameters of STAR-Fusion. Raw chimeric read data also showed no evidence of CIC-DUX4 fusion reads (Supplemental Table 2) nor did manual aligned read extraction via "grep" (Panagopoulos et al. 2014). We expanded the search to any reads supporting a fusion between Chromosome 4 (location of DUX4) and Chromosome 19 (location of CIC). All t $(4,19)$ translocation support reads were annotated with biomaRt and GeneLoc (Tables 5 and 6). GH04J119298, a gene promoter annotated in GeneLoc, was proximal to the right breakpoint in several reads. Most other annotated gene pairs bookend the right fusion breakpoint. For example, the breakpoint (190069535) is between GC04P190068 (190068071190069067) and DUX4L7 (190071232-190072516). Additionally, all $t(4,19)$ fusion reads included CIC as a fusion partner. Although the canonical CIC-DUX4 fusion was not found, support exists for potential CIC-DUX4L7 fusion. These findings point to the potential need to develop a FISH panel for CIC-DUX variant fusions.

As we lacked RNA sequencing from normal adjacent or contralateral tissue, we used the median TPM values of a cohort of skeletal muscle tissues $(n=564)$ in the Genotype-Tissue Expression (GTEx) project as a comparison. Previous studies have seen up-regulation of genes in the PEA3 family in CIC-DUX4 sarcomas (Kawamura-Saito et al. 2006; Kao et al. 2017). In both the pretreatment and post-treatment samples, we found twofold higher expression levels of PEA3 family genes, such as BAX, CCND2, ETV1, ETV4, ETV5, FGF8, GBX2, ICAM1, MMP2/9, NOTCH1, PLAU, PTK2, SNAI1/2, SPRED2, SPRY2, SPRY4, and TWIST1, relative to the population of skeletal muscle tissues in the GTEx project (Table 7). In the pretreatment sample only, we found MMP1/7 and PTGS2 to have twofold higher expression levels and in the post-treatment sample we found NOTCH4 and SPP1 to have twofold higher expression levels (Table 7). We found twofold higher expression levels of eEF1A1 (13,778 TPM pretreatment and 12,775 TPM post-treatment) and RPL32 (4915 TPM pretreatment and 4168 TPM post-treatment) (Table 8; Figs. 3 and 4). Moreover, eEF1A1 was found to have twofold higher expression compared to the median expression level of all nontumorous tissues sequenced in the GTEx project. Further, for most samples in our cohort, eEF1A1 expression of non-rhabdomyosarcomas soft tissue sarcomas (NRSTS) and rhabdomyosarcomas was found to be higher than that of nontumorous tissues (Fig. 5). Embryonal rhabdomyosarcoma (ERMS) cell lines Ruch2, Ruch3, SCMC-RMS, and RMS-YM, were found to have lower eEF1A1 expression potentially because of a difference in sequencing platforms used. Thus, further investigation into the role of eEF1A1 in multiple childhood cancers is warranted.

To validate the expression levels seen in RNA sequencing across several childhood cancers we immunoblotted for EEF1A1 in NRSTS $(n=4)$ and RMS $(n=7)$ biopsy samples (Fig. 6A) and cell lines (Fig. 6B). In the absence of CIC-rearranged sarcoma (CRS) biopsy samples, UPS 


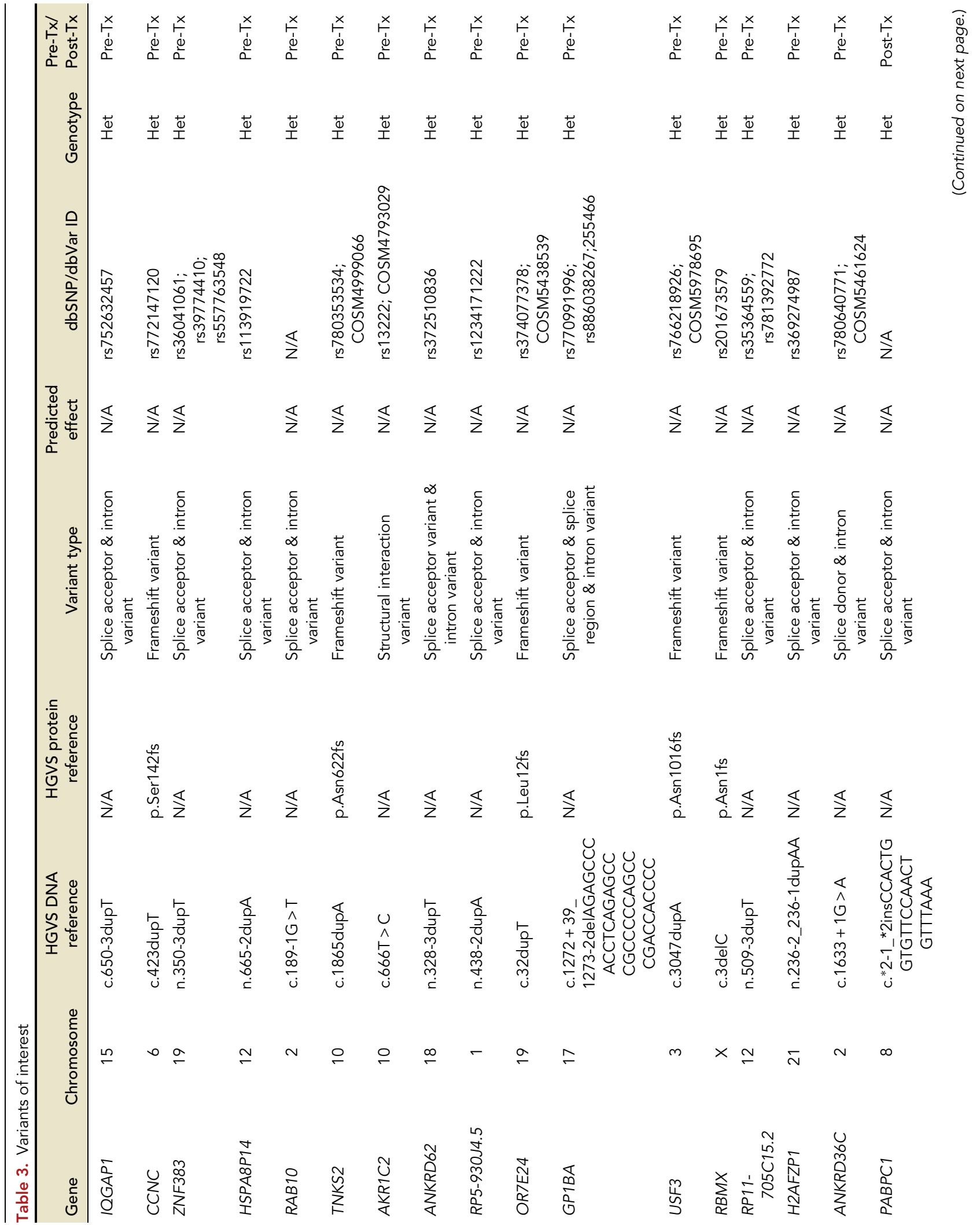




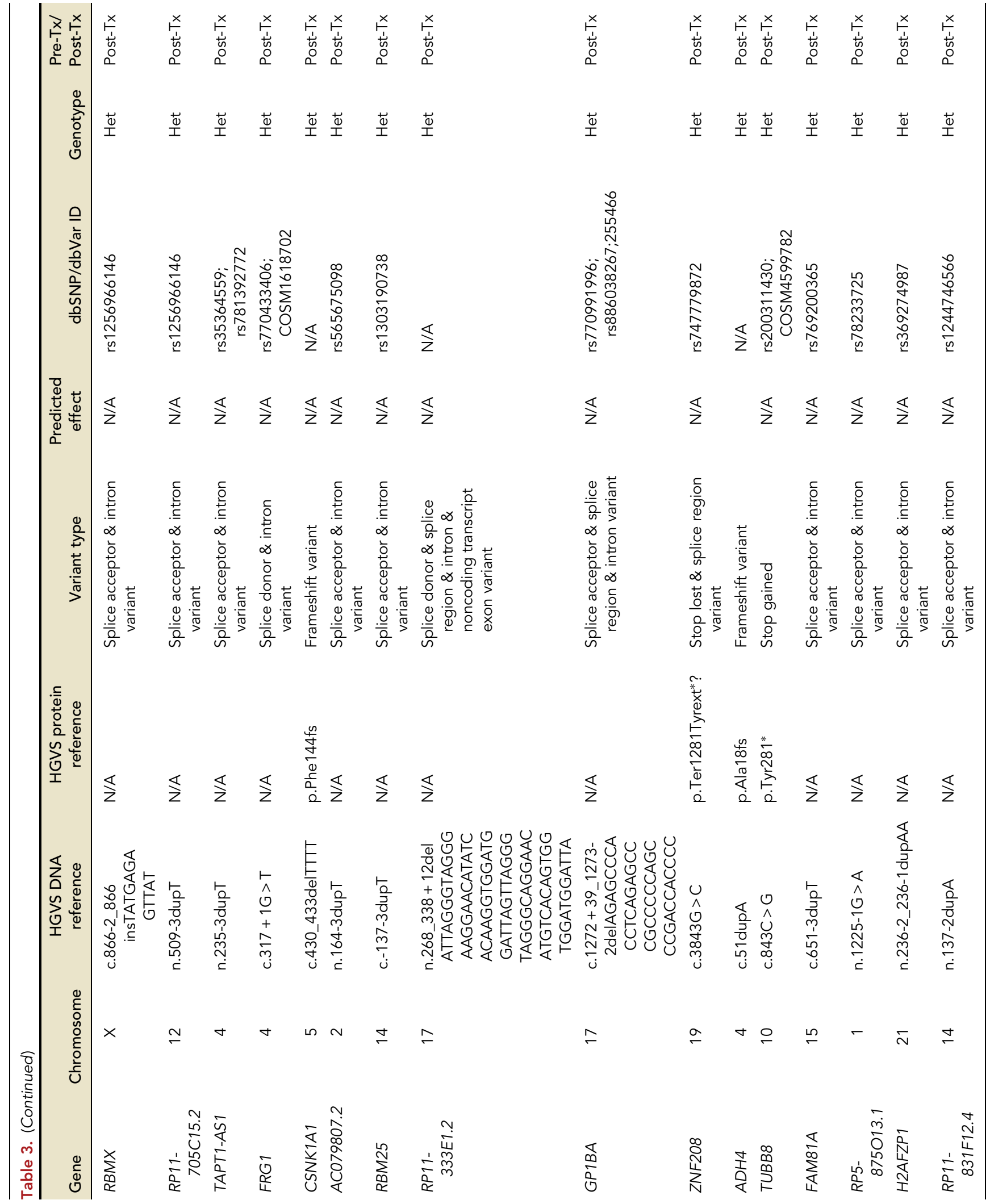




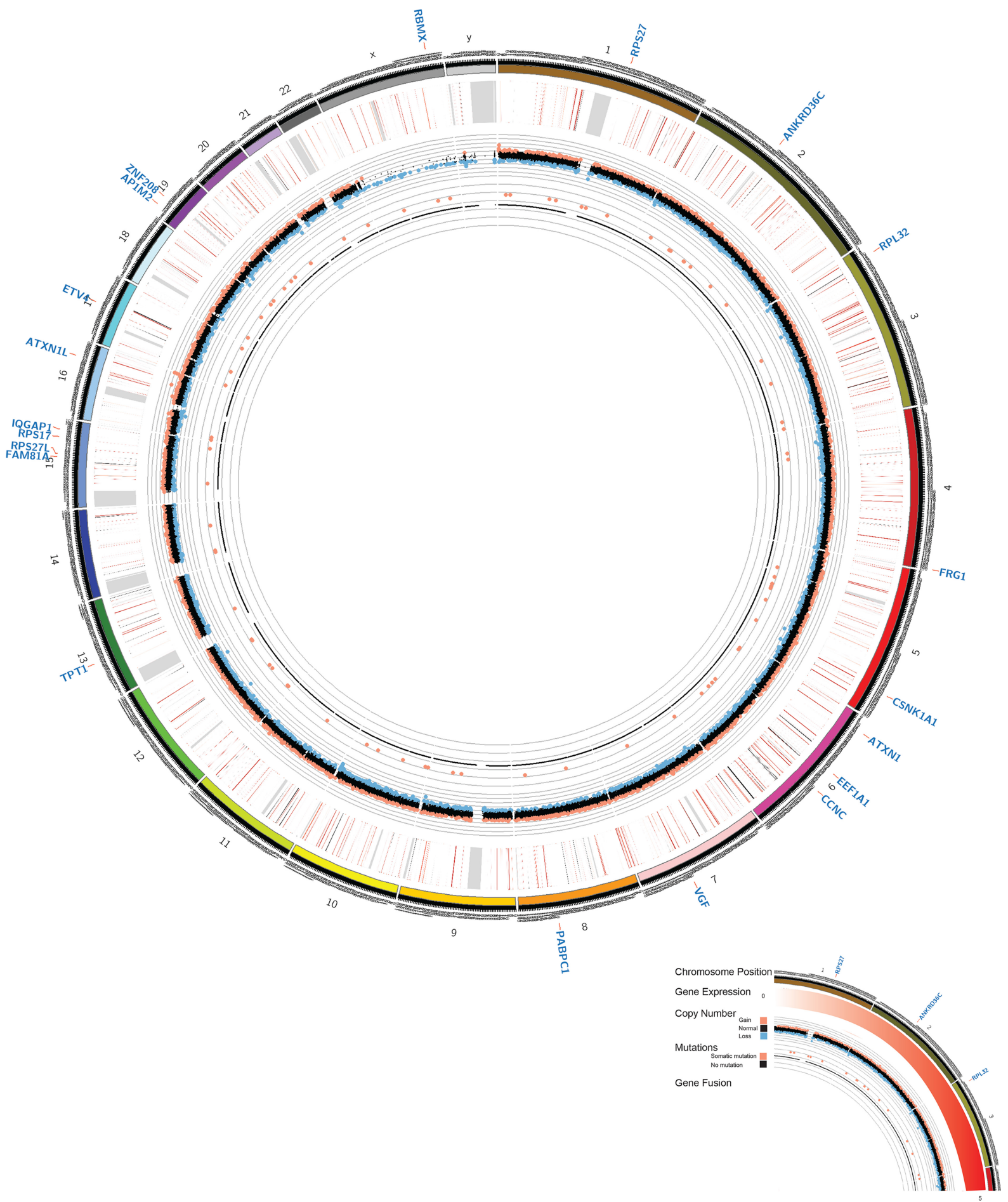

Figure 3. Circos plot of pretreatment tumor sample. Circos representation of genomic and transcriptomic data, including potential gene fusions (circle center), mutations/indels (innermost ring), copy-number variations (middle ring), gene expression (outer ring), and genes of interest determined by involvement in potential gene fusions of being mutated, amplified, and overexpressed. Key findings include the overexpression of EEF1A1 and somatic mutations, such as duplication in IQGAP1, a frameshift in CCNC, and amplification at ATXN1L. 


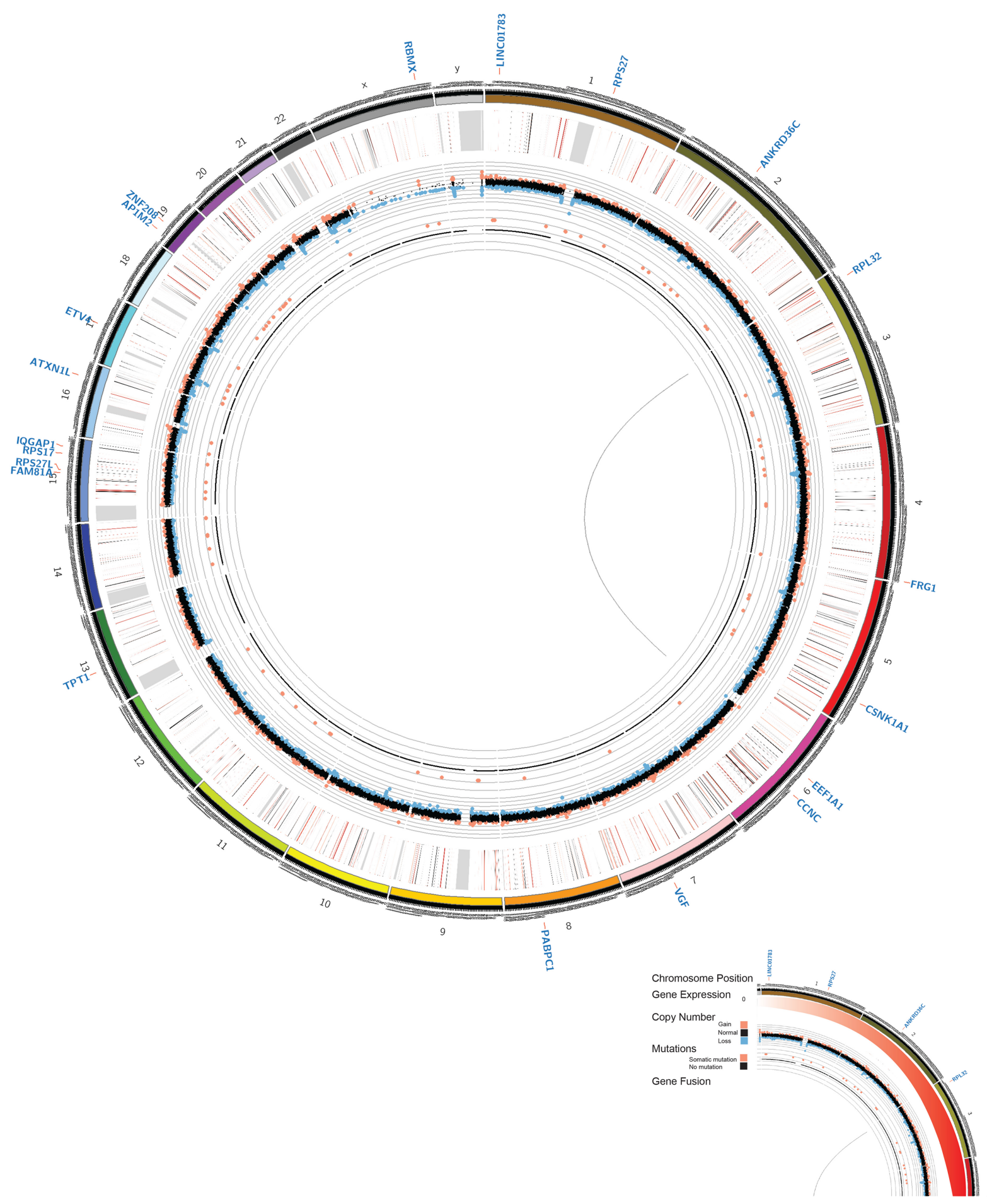

Figure 4. Circos plot of the post-treatment tumor sample. Circos representation of genomic and transcriptomic data, including potential gene fusions (circle center), mutations/indels (innermost ring), copy-number variations (middle ring), gene expression (outer ring), and genes of interest determined by involvement in potential gene fusions of being mutated, amplified, and overexpressed. Key findings include the overexpression of VGF, AP1M2, and ETV4 in post-treatment compared to both population normal and pretreatment RNA sequencing. Other key somatic mutations include an insertion in the PABPC gene, a duplication in the TAPT1AS1 gene, a splice-donor site in the FRG1 gene, a frameshift deletion in the CSNK1A1 gene, a stop codon lost in the ZNF208 gene, a duplication in the FAM81A gene, and a splice-acceptor site in the LINC01783 gene. The EEF1A1-RPL32 fusion gene was also identified with a left breakpoint at Chr 6:73519384 and a right breakpoint at Chr 3:12839458. 
Table 4. Somatic mutation burden

\begin{tabular}{lcc}
\hline & Pretreatment & Post-treatment \\
\hline High & 16 & 17 \\
Moderate & 75 & 73 \\
Low & 405 & 413 \\
\hline
\end{tabular}

and epithelioid sarcoma (ES) biopsy samples and cell lines were used to represent NRSTS cases (Tables 9 and 10). RMS samples and the ES sample both had expression similar to or higher than normal skeletal muscle tissues and the human skeletal muscle myoblast (HSMM) cell line. The two UPS biopsy samples demonstrated lower expression than normal skeletal muscle, whereas the UPS cell line exhibited a similar or slightly lower expression level compared to HSMM. More NRSTS samples-in particular, more CRS samples-should be examined to provide a more accurate representation of the disease.

To further classify the molecular features of the pre- and post-treatment tumors in the context of sarcoma, we used unsupervised clustering to determine RMS and NRSTS endotypes (subgroups of samples with distinct pathobiological mechanisms). We used RNA and DNA from RMS and NRSTS patient samples $(n=53)$, patient-derived xenografts $(n=6)$, and cell lines $(n=16)$. In hierarchical clustering of both somatic mutations and gene expression, the patient clusters closest to CTG-1542, a PDX model of metastatic ERMS (Fig. 7). Most cell lines with both RNA-seq and WES available developed their own cluster, most likely because of a lack of a matched-normal sample resulting in several false positive mutations. Interestingly, in the hierarchical clustering of gene expression alone, EEF1A1 was found to have the highest gene expression level across the entire cohort (Fig. 8). Hierarchical clustering of gene expression alone identified CLF-PED-015-T (pediatric UPS) (Hong et al. 2016) to be in the endotype of the patient's pre- and post-treatment samples (Fig. 8). Parenthetically, if CRS cell lines had been available, CLF-PED-015-T may have been less closely related-pointing to the need to develop CRS cell lines. Given the resources available to us, we analyzed the tumor and matched normal sequencing data from CLF-PED-015-T using the same analysis pipeline and methods as the patient. We found eEF1A1 to also have twofold higher expression (9891 TPM) in the primary cell line compared to the population of skeletal muscle tissues from GTEx

\begin{tabular}{|c|c|c|c|c|c|c|c|c|}
\hline $\begin{array}{l}\text { Left } \\
\text { Chr }\end{array}$ & $\begin{array}{l}\text { Left } \\
\text { break }\end{array}$ & $\begin{array}{l}\text { Left } \\
\text { strand }\end{array}$ & Gene & $\begin{array}{l}\text { Right } \\
\text { Chr }\end{array}$ & $\begin{array}{l}\text { Right } \\
\text { break }\end{array}$ & $\begin{array}{l}\text { Right } \\
\text { strand }\end{array}$ & Gene (Left) & $\begin{array}{c}\text { Gene } \\
\text { (Right) }\end{array}$ \\
\hline Chr 19 & 42290692 & - & $\mathrm{ClC}$ & Chr 4 & 119300386 & + & GH04J119298 & \\
\hline Chr 19 & 42294904 & - & $\mathrm{ClC}$ & Chr 4 & 190069353 & - & GC04P190068 & DUX4L7 \\
\hline Chr 19 & 42294904 & - & $\mathrm{ClC}$ & Chr 4 & 190069353 & - & GC04P190068 & DUX4L7 \\
\hline Chr 19 & 42294904 & - & $\mathrm{ClC}$ & Chr 4 & 190069353 & - & GC04P190068 & DUX4L7 \\
\hline Chr 19 & 42294904 & - & $\mathrm{ClC}$ & Chr 4 & 190069353 & - & GC04P190068 & DUX4L7 \\
\hline Chr 19 & 42294904 & - & $\mathrm{ClC}$ & Chr 4 & 190069353 & - & GC04P190068 & DUX4L7 \\
\hline Chr 19 & 42294904 & - & $\mathrm{CIC}$ & Chr 4 & 190069353 & - & GC04P190068 & DUX4L7 \\
\hline Chr 19 & 42294904 & - & $\mathrm{ClC}$ & Chr 4 & 190069353 & - & GC04P190068 & DUX4L7 \\
\hline Chr 19 & 42294905 & - & $\mathrm{ClC}$ & Chr 4 & 190069356 & - & GC04P190068 & DUX4L7 \\
\hline Chr 19 & 42294905 & - & $\mathrm{ClC}$ & Chr 4 & 190069356 & - & GC04P190068 & DUX4L7 \\
\hline Chr 19 & 42294736 & - & $\mathrm{ClC}$ & Chr 4 & 190092456 & - & GC04P190068 & DUX4L7 \\
\hline Chr 19 & 42294736 & - & $\mathrm{ClC}$ & Chr 4 & 190092456 & - & GC04P190068 & DUX4L7 \\
\hline
\end{tabular}




\begin{tabular}{|c|c|c|c|c|c|c|c|c|}
\hline $\begin{array}{l}\text { Left } \\
\text { Chr }\end{array}$ & Left break & $\begin{array}{l}\text { Left } \\
\text { strand }\end{array}$ & Gene & $\begin{array}{l}\text { Right } \\
\text { Chr }\end{array}$ & Right break & $\begin{array}{l}\text { Right } \\
\text { strand }\end{array}$ & Gene (Left) & $\begin{array}{c}\text { Gene } \\
\text { (Right) }\end{array}$ \\
\hline Chr 19 & $42,293,119$ & - & $\mathrm{ClC}$ & Chr 4 & $1,169,692$ & + & SPON2 & \\
\hline Chr 19 & $42,273,290$ & - & $\mathrm{CIC}$ & Chr 4 & $1,172,702$ & + & SPON2 & \\
\hline Chr 19 & $42,273,290$ & - & $\mathrm{ClC}$ & Chr 4 & $1,172,702$ & + & SPON2 & \\
\hline Chr 19 & $42,289,208$ & - & $\mathrm{ClC}$ & Chr 4 & $41,689,572$ & - & LIMCH1 & \\
\hline Chr 19 & $42,291,733$ & - & $\mathrm{CIC}$ & Chr 4 & $42,401,468$ & - & SHISA3 & \\
\hline Chr 19 & $42,294,683$ & - & $\mathrm{ClC}$ & Chr 4 & $48,512,599$ & + & FRYL & \\
\hline Chr 19 & $42,291,411$ & - & $\mathrm{ClC}$ & Chr 4 & $87,494,241$ & + & SPARCL1 & \\
\hline Chr 19 & $42,291,411$ & - & $\mathrm{ClC}$ & Chr 4 & $87,494,241$ & + & SPARCL1 & \\
\hline Chr 19 & $42,286,777$ & - & $\mathrm{ClC}$ & Chr 4 & $113,453,541$ & + & CAMK2D & \\
\hline Chr 19 & $42,293,701$ & - & $\mathrm{ClC}$ & Chr 4 & $56,332,297$ & + & RNA5SP162 & AASDH \\
\hline Chr 19 & $42,287,123$ & - & $\mathrm{ClC}$ & Chr 4 & $121,696,592$ & + & ANXA5 & \\
\hline Chr 19 & $42,287,123$ & - & $\mathrm{ClC}$ & Chr 4 & $121,696,592$ & + & ANXA5 & \\
\hline Chr 19 & $42,294,062$ & - & $\mathrm{ClC}$ & Chr 4 & $153,596,272$ & - & TMEM131L & \\
\hline Chr 19 & $42,294,877$ & - & $\mathrm{ClC}$ & Chr 4 & $190,069,353$ & - & GC04P190068 & DUX4L7 \\
\hline Chr 19 & $42,294,904$ & - & $\mathrm{ClC}$ & Chr 4 & $190,069,353$ & - & GC04P190068 & DUX4L7 \\
\hline Chr 19 & $42,294,904$ & - & $\mathrm{ClC}$ & Chr 4 & $190,069,353$ & - & GC04P190068 & DUX4L7 \\
\hline Chr 19 & $42,294,904$ & - & $\mathrm{ClC}$ & Chr 4 & $190,069,353$ & - & GC04P190068 & DUX4L7 \\
\hline Chr 19 & $42,294,904$ & - & $\mathrm{ClC}$ & Chr 4 & $190,069,353$ & - & GC04P190068 & DUX4L7 \\
\hline Chr 19 & $42,294,904$ & - & $\mathrm{ClC}$ & Chr 4 & $190,069,353$ & - & GC04P190068 & DUX4L7 \\
\hline Chr 19 & $42,294,904$ & - & $\mathrm{ClC}$ & Chr 4 & $190,069,353$ & - & GC04P190068 & DUX4L7 \\
\hline Chr 19 & $42,294,905$ & - & $\mathrm{ClC}$ & Chr 4 & $190,069,359$ & - & GC04P190068 & DUX4L7 \\
\hline
\end{tabular}

Portal ( $n=564$, median TPM $=207$ ) (Table 11). Additionally, we saw overexpression of ETV4 (1608 TPM), which is frequently used as a diagnostic tool for CRS cases (Hung et al. 2016). Given the biological similarities, immunohistochemistry to test for the presence of diffuse DUX4 nuclear staining in CLF-PED-015-T could be warranted. Because the patient's pre- and post-treatment samples were found to have similar molecular features to CLFPED-015-T and a cell line has not been developed from the patient's tumor sample, CLFPED-015-T might serve as a surrogate model in the absence of any CRS cell lines.

\section{DISCUSSION}

In this case study, pre- and post-treatment tumor DNA whole-genome samples were sequenced and analyzed against matched normal samples, as well as RNA sequencing of pre- and post-treatment tumor tissue. Sequencing revealed overexpression of the eEF1A1 translational factor, which is of special interest in this case. eEF1A1, an isoform of the a subunit of the eukaryotic Elongation Factor 1, is commonly overexpressed in human tumors and has oncogenic properties that favor tumor cell proliferation and inhibit apoptosis. eEF1A1 is reported to be a negative regulator p53 and p73 and leading to chemoresistance (Blanch et al. 2013), and the ubiquitin-like protein FAT10 has been shown to bind eEF1A1 which stabilizes the expression of eEF1A1 in favor of tumor cell proliferation (Liu et al. 2016).

The isoform eEF1A1 shares $96 \%$ homology with the isoform eEF1A2, the primary target of plitidepsin (dehydrodidemnin B/Aplidin, PharmaMar), an antitumor agent of marine origin 
Table 7. Expression of genes in the PEA 3 in the pretreatment and post-treatment tumor samples compared to the collection of skeletal muscle tissues in the Genotype-Tissue Expression (GTEx) project

\begin{tabular}{|c|c|c|c|c|c|}
\hline \multicolumn{3}{|c|}{ Pretreatment } & \multicolumn{3}{|c|}{ Post-treatment } \\
\hline Gene & TPM & $\mathrm{TU} /(\mathrm{NM}+0.01)$ & Gene & TPM & $\mathrm{TU} /(\mathrm{NM}+0.01)$ \\
\hline$B A X$ & 459.06 & 43.36892 & $B A X$ & 315.43 & 29.79972 \\
\hline CCND2 & 140.65 & 110.6174 & CCND2 & 260.96 & 205.2379 \\
\hline ETV1 & 326.83 & 1410.574 & ETV1 & 396.14 & 1709.711 \\
\hline ETV4 & 1152.69 & 5699.333 & ETV4 & 1784.83 & 8824.87 \\
\hline ETV5 & 568.37 & 94.06984 & ETV5 & 675.87 & 111.862 \\
\hline FGF8 & 0.1 & 2.242152 & FGF8 & 0 & 0 \\
\hline GBX2 & 2.36 & 236 & GBX2 & 16.65 & 1665 \\
\hline GPR2O & 0 & 0 & GPR20 & 0.21 & 0.854875 \\
\hline ICAM1 & 83.98 & 15.41483 & ICAM1 & 16.98 & 3.11674 \\
\hline MMP1 & 0.29 & 9.392713 & MMP1 & 0.06 & 1.94332 \\
\hline MMP2 & 755.74 & 32.26211 & MMP2 & 1828.44 & 78.05507 \\
\hline MMP3 & 0 & 0 & MMP3 & 0.06 & 0.015998 \\
\hline MMP7 & 0.32 & 5.978515 & MMP7 & 0.06 & 1.120972 \\
\hline MMP9 & 35.7 & 101.4493 & MMP9 & 128.44 & 364.9901 \\
\hline NOTCH1 & 48.51 & 12.36554 & NOTCH1 & 49.9 & 12.71986 \\
\hline NOTCH4 & 4.47 & 0.568305 & $\mathrm{NOTCH} 4$ & 19.57 & 2.488081 \\
\hline PLAU & 11.61 & 2.053958 & PLAU & 12.76 & 2.257408 \\
\hline POU5F1 & 0.32 & 0.727769 & POU5F1 & 0.33 & 0.750512 \\
\hline PTGS2 & 7.42 & 60.2762 & PTGS2 & 0.04 & 0.324939 \\
\hline PTK2 & 57.92 & 6.136568 & PTK2 & 65.75 & 6.966149 \\
\hline SNAl1 & 3.61 & 2.68003 & SNAI1 & 4.08 & 3.028953 \\
\hline SNAI2 & 14.04 & 5.546119 & SNAI2 & 20.34 & 8.034762 \\
\hline SPP1 & 523.48 & 1010.482 & SPP1 & 72.17 & 139.3109 \\
\hline SPRED2 & 92.69 & 21.17177 & SPRED2 & 102.88 & 23.49931 \\
\hline SPRY2 & 62.31 & 3.382736 & SPRY2 & 208.45 & 11.3165 \\
\hline SPRY4 & 145.03 & 44.75544 & SPRY4 & 122.09 & 37.67628 \\
\hline TWIST1 & 27.41 & 72.56122 & TWIST1 & 21.84 & 57.81602 \\
\hline VEGFA & 41.43 & 0.314913 & VEGFA & 37.41 & 0.284357 \\
\hline ZEB1 & 25.08 & 0.903784 & ZEB1 & 24.26 & 0.874234 \\
\hline
\end{tabular}

(Losada et al. 2016). eEF1A isoforms were the only proteins detected as capable of binding plitidepsin, and sensitivity was dependent on eEF1A2 levels. Plitidepsin has completed phase III clinical trials for multiple myeloma, but more studies are necessary to validate the interaction between plitidepsin and eEF1A1 and the oncogenic properties and effectiveness of plitidepsin.

A major limitation of this work is the absence of a patient-derived cell model for phenotypic screening of plitidepsin and other agents of interest. Coclinical trials allow for parallel or sequential screening of human primary tumors in mouse models. Particularly for cases where there is little clinically actionable molecular information, development of coclinical methods for phenotypic screening of primary tumors samples using "endotype" surrogate cell lines would help us understand the tumors' response to therapeutic agents. Our work presents evidence of a potential therapeutic target, advances our knowledge of an understudied 
COLD SPRING HARBOR Molecular Case Studies
Translation factor eEF1A1 overexpression in a CIC-rearranged sarcoma case
Table 8. Summary of the expression levels of standout genes from pretreatment and post-treatment tumor samples compared to the collection of skeletal muscle tissues in the Genotype-Tissue Expression (GTEx) project

\begin{tabular}{llllll}
\hline & \multicolumn{2}{c}{ Pretreatment } & & \multicolumn{2}{c}{ Post-treatment } \\
\cline { 2 - 3 } \cline { 5 - 6 } Gene & TPM & TU/(NM+0.01) & & TPM & TU/(NM + 0.01) \\
\hline eEF1A1 & 13,778 & 66 & & 12,775 & 62 \\
eEF1A2 & 0.17 & $6.82 \times 10^{-5}$ & & 3.2 & 0.001284 \\
RPL32 & 4,915 & 15 & & 4,168 & 13 \\
VGF & 5,381 & 94,680 & & 7,304 & 193,120 \\
AP1M2 & 1,519 & 34,303 & & 2,402 & 54,244 \\
ETV4 & 1,153 & 5,699 & & 1,785 & 8,825 \\
\hline
\end{tabular}

set of childhood cancers (CIC-rearranged sarcomas), and highlights the importance of future preclinical studies and cell line/mouse model development for CRS.

Clinically, at least three of the four pathologists whom did not identify CRS in this patient had training in and are well-published in sarcomas. However, CRS is rare and has only recently been identified as their own distinct subgroup, making them challenging to identify. Given that CRS is a new entity that can go unrecognized, increased scrutiny of round cell histology undifferentiated sarcomas and development of confirmatory tools such as methylation profiling or FISH for variant CIC-DUX4-like fusions appears warranted.

Previous studies have also had difficulty identifying the CIC-DUX4 fusion gene using several fusion callers (Panagopoulos et al. 2014). A possible explanation for the failure in the fusion callers' ability to identify the CIC-DUX4 fusion gene is the large number of DUX4 pseudogenes with very similar sequences (e.g., concordance in 1283 of the first 1285 nucleotides in DUX4 and DUX4L7, which represents 1285 of the 1553 base pairs of DUX4 Exon 1, noted to be the location of most CIC-DUX4 fusions). Despite several fusion callers with multiple calling parameters failing to call CIC-DUX4, other fusion calling tools may be able to identify CIC-DUX4. A multifusion caller pipeline may be warranted to capture this challenging gene fusion, but consensus calling approaches will still be limited by the challenge in calling CIC-DUX4.

Here, we have demonstrated a case of an 11-yr-old male presented with a soft tissue mass that was later identified to be CRS. Using RNA sequencing from pretreatment and post-treatment tumor samples we have found an abundance of $P E A 3$ family genes overexpressed, as well as eEF1A1 translational factor. Given the overexpression of eEF1A1 in this aggressive and severe NRSTS (CIC-rearranged sarcoma) the availability of plitidepsin as an eEF1A1/2 anti-tumor agent is potentially interesting. Our observations could warrant further in vivo preclinical studies to test whether eEF1A over-expression in CRS and other NRSTS implies sensitivity to plitidepsin. Such studies would likely be predicated on detection of eEF1A1/2 over-expression observed across a meaningfully large NRSTS subpopulation.

\section{METHODS}

\section{Cell Lines}

RMS559, CW9019, CCA, RD, Hs729T, COG-R-486h, and SMS-CTR were grown in Dulbecco's Modified Eagle Medium (DMEM) supplemented with $10 \%$ fetal bovine serum (FBS) and 1\% penicillin/streptomycin. COG-R-486h was maintained in hypoxic conditions. 
EEF1A1
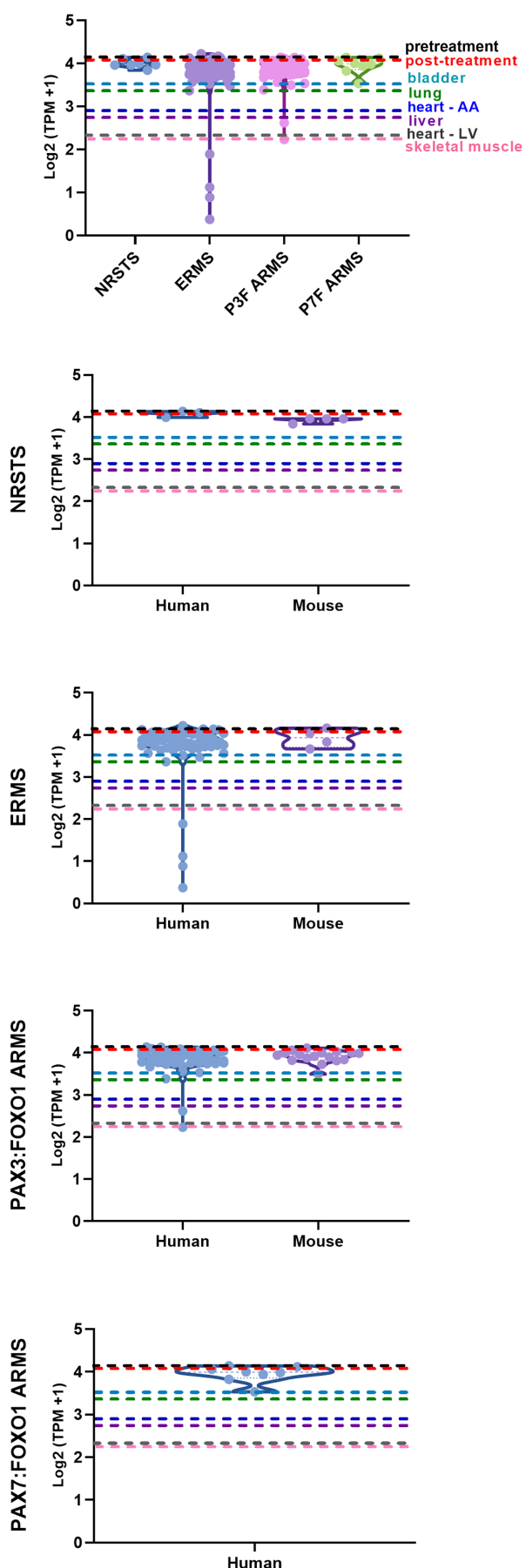

EEF1A2
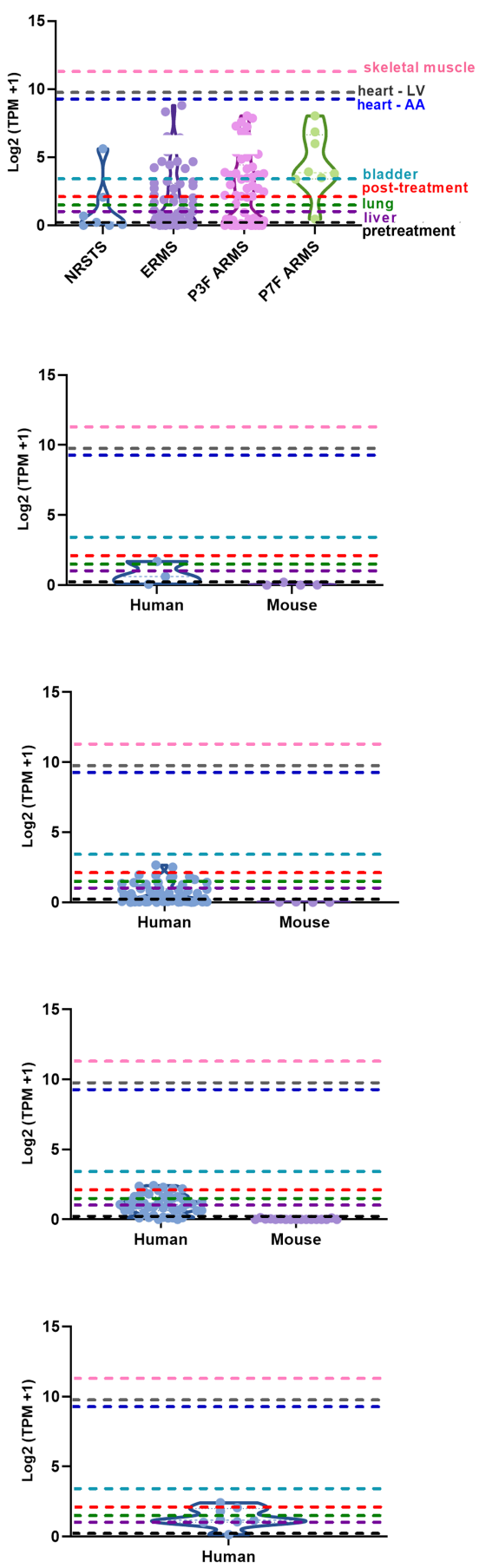

Figure 5. Gene expression of EEF1A1 and EEF1A2 in NRSTS, ERMS, PAX3:FOXO1 ARMS, and PAX7:FOXO1 ARMS. Violin plots of the expression level of EEF1A1 and EEF1A2 in human biopsies, patient-derived xenografts, and cell lines (blue) and genetically engineered mouse models (purple). The median gene expression level from the bladder $(n=11)$, lung $(n=427)$, atrial appendage (AA) $(n=297)$, liver $(n=175)$, left ventricle (LV) ( $n$ $=303)$, and skeletal muscle $(n=564)$ tissues shown by the light blue, green, dark blue, purple, gray, and pink dashed lines, respectively. The expression level of EEF1A1 and EEF1A2 pretreatment and post-treatment is shown by the black and red dashed lines, respectively. 
COLD SPRING HARBOR Molecular Case Studies
Translation factor eEF1A1 overexpression in a ClC-rearranged sarcoma case
A
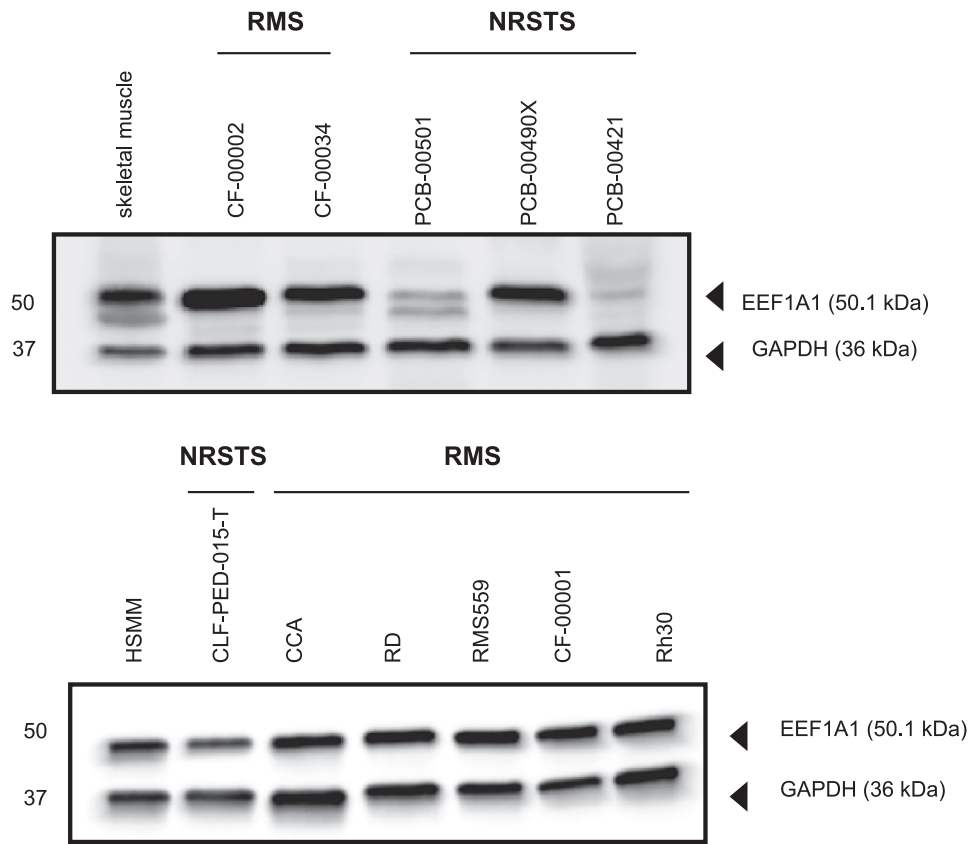

Figure 6. EEF1A1 is overexpressed in UPS and ARMS tumors compared to normal skeletal muscle. (A) Western blotting was carried out on nonrhabdomyosarcoma soft tissue sarcoma (NRSTS) biopsies (PCB501, PCB490X, and PCB421), fusion-negative alveolar rhabdomyosarcoma (ARMS) biopsy (CF-00449), PAX7: FOXO1 fusion ARMS biopsy (CF-00002), PAX3:FOXO1 ARMS biopsy (CF-00034), and normal muscle samples (skeletal muscle biopsy CF-00101). (B) Western blotting was also carried out on a NRSTS cell line (CLF-PED015-T), embryonal rhabdomyosarcoma (ERMS) cell lines (CCA, RD, and RMS559), a PAX7:FOXO1 ARMS cell line (CF-00001), a PAX3:FOXO1 ARMS cell line (Rh30), and a human skeletal muscle myoblast cell line (HSMM). Cell lines are described in Table 10.

HSMM, SkMc, RH5, Rh18, CLF-PED-015T, and RMS13 were grown in Roswell Park Memorial Institute (RPMI) 1640 medium supplemented with 10\% FBS and 1\% penicillin/ streptomycin.

\section{Human Biopsy Samples}

All human samples were provided through our CuReFAST tumor registry with the approval of the Institutional Review Board.

Table 9. Demographics of biopsy samples

\begin{tabular}{|c|c|c|c|c|c|}
\hline $\begin{array}{l}\text { Sample } \\
\text { name }\end{array}$ & Presumptive diagnosis & Pathological diagnosis & Fusion status & Gender & Age (yr) \\
\hline PCB-00501 & $\begin{array}{l}\text { Undifferentiated pleomorphic } \\
\text { sarcoma }\end{array}$ & $\begin{array}{l}\text { High-grade pleomorphic sarcoma, not otherwise } \\
\text { specified }\end{array}$ & Fusion negative & Male & 67 \\
\hline PCB-00490X & Epithelioid sarcoma & $\begin{array}{l}\text { Epithelioid sarcoma, infiltrating skeletal muscle } \\
\text { and fibroadipose tissue }\end{array}$ & Fusion negative & Female & 22 \\
\hline PCB-00421 & $\begin{array}{l}\text { Undifferentiated pleomorphic } \\
\text { sarcoma }\end{array}$ & High-grade sarcoma with extensive necrosis & Fusion negative & Unknown & Unknown \\
\hline CF-00002 & ARMS & Unknown & $\mathrm{PAX7:FOXO1}$ & Female & 6 \\
\hline CF-00034 & ARMS & ARMS & PAX3:FOXO1 & Male & 17 \\
\hline
\end{tabular}


COLD SPRING HARBOR Molecular Case Studies
Translation factor eEF1A1 overexpression in a CIC-rearranged sarcoma case

\begin{tabular}{lclll}
\hline Table 10. Demographics of cell lines & & & \\
\hline Sample name & \multicolumn{1}{c}{ Diagnosis } & Fusion status & Gender & Age (yr) \\
\hline CLF-PED-015-T & $\begin{array}{c}\text { Undifferentiated } \\
\text { pleomorphic sarcoma }\end{array}$ & Fusion negative & Unknown & Unknown \\
CCA & ERMS & Fusion negative & Male & 8 \\
RD & ERMS & Fusion negative & Female & 7 \\
RMS559 & ERMS & Fusion negative & Male & Unknown \\
CF-00001 & ARMS & PAX3:FOXO1 & Male & 1 \\
Rh30 & ARMS & PAX3:FOXO1 & Male & 17 \\
\hline
\end{tabular}

\section{Whole-Genome Sequencing}

Tumor and matched normal genome sequencing data were analyzed for the presence of somatic point mutation, somatic functional and structural mutations, potential germline mutations, polynucleotide insertions and deletions, and gene CNVs. Sequence reads were aligned to the GRCh38 human reference genome and somatic mutations and indels were called using Genome Analysis Toolkit (GATK) Version 4.0 with stricter calling criterion (Tumor logarithm of odds [TLOD] scores >6.3). Gene CNVs were identified using SAMtools and VarScan2 quantified as a log ratio of tumor copy to normal copy. Regions with a log ratio $>0.60$ were called as gained, regions with a log ratio $<-0.60$ were called as lost. Genes overlapping gained or lost regions by at least $15 \%$ of the gene's genomic region were called as gained or lost, respectively. Sequencing coverage of the pretreatment and post-treatment samples are noted in Supplemental Table 3.

\section{RNA Sequencing}

RNA sequencing data was analyzed for gene expression and gene fusion events. Transcriptome data was aligned to the GRCh38 human reference genome. Normalized gene expression was quantified using RSEM. Non-tumor gene expression data was not provided, thus region-specific unmatched skeletal muscle tissue gene expression data was accessed from the GTEx project to serve as a population normal and to identify underexpressed and overexpressed genes. Gene fusion events were identified using STAR-Fusion. Sequencing coverage of the pretreatment and post-treatment samples are noted in Supplemental Table 3.

\section{Circos Plots}

Circos plots to visualize the collected genomics and transcriptomics data were generated using tumor DNA genome, normal DNA genome, and tumor RNA sequencing data. Genes identified as bearing variants, having increased gene copy number, and overexpressed compared to population normal are identified in blue around both circos plots (Figs. 3 and 4, pretreatment and post-treatment analyses). Also identified in blue are genes involved in identified gene fusion events and genes identified as being of interest because of overarching genetic and transcriptomic features in the tumor samples.

\section{Hierarchical Sequencing Analysis}

RNA and DNA sequencing of cell lines, patient-derived xenograft (PDX) mouse models, and patient's biopsy samples were collected from numerous sources including St. Jude Children's Research Hospital, the Jackson Laboratory, Champions Oncology (COG), Gene Expression Omnibus (GEO), the National Cancer Institute (NCl), and through our IRB- 


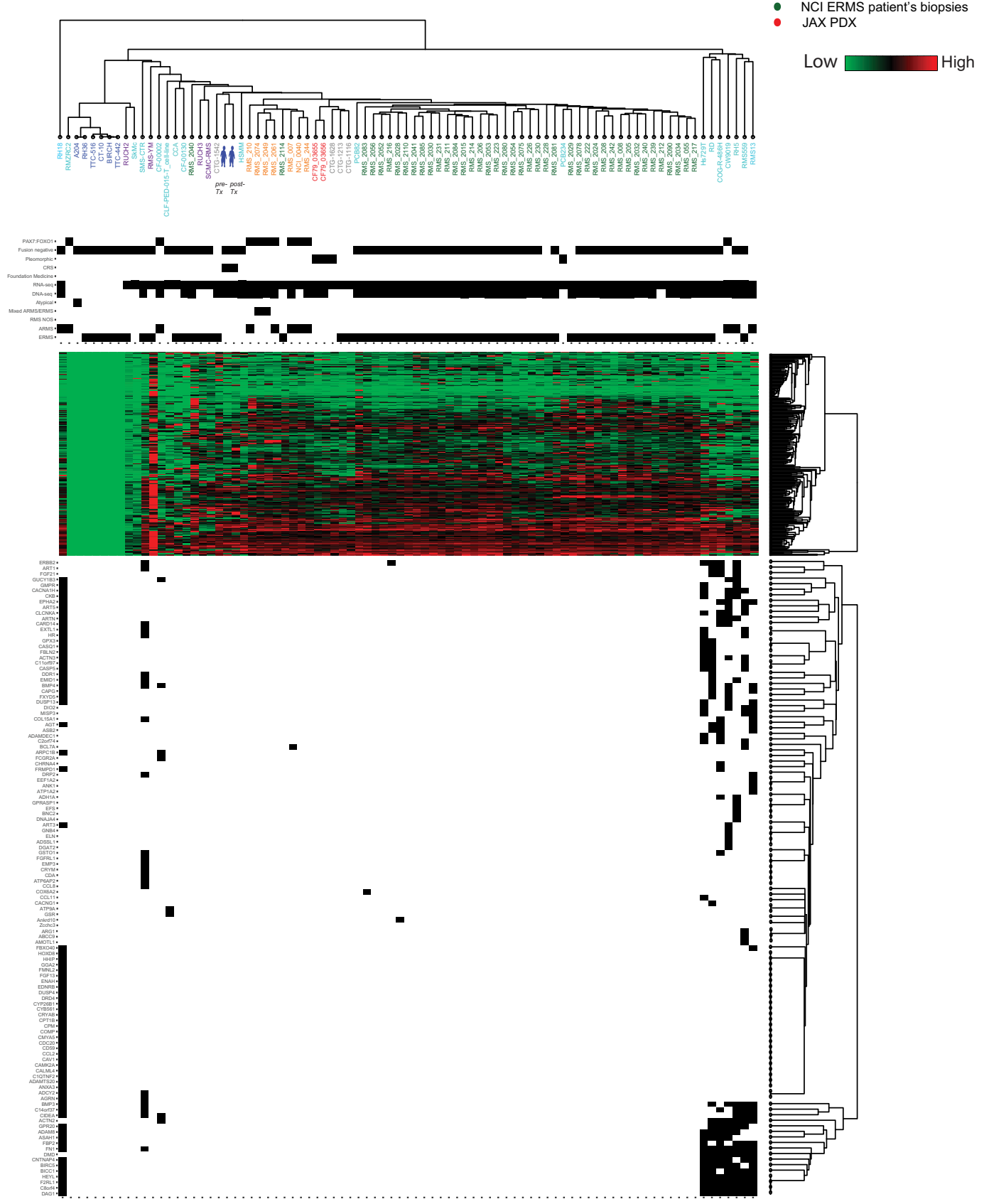

Figure 7. Index case endotype. Unsupervised clustering of embryonal rhabdomyosarcoma (ERMS) and nonrhabdomyosarcoma soft tissue sarcoma (NRSTS) samples with alveolar rhabdomyosarcoma (ARMS) samples harboring the PAX7:FOXO1 fusion gene as a control. Clustering was performed using RNA and DNA sequencing from tumor samples from our IRB-approved CuReFAST initiative and cell lines (light blue), cell lines from Gene Expression Omnibus (GEO) (purple), patient-derived xenograft (PDX) mouse models from Champions Oncology (gray), cell lines from St. Jude Children's Research Hospital (dark blue), ARMS patient's biopsies from National Cancer Institute (orange), ERMS patient's biopsies from NCl (green), PDX mouse models from the Jackson Laboratory (red), and the patient's tumor sample (dark blue avatar) before treatment and after treatment. The legend below marks samples' fusion status, diagnosis, and whether RNA and/or DNA sequencing was included in the dendrogram. Unsupervised clustering was performed on the data within the legend (right). The matrix below has a sample in each column and gene in each row. The scale bar shows the relative gene expression per gene with red being the highest expression and green being the lowest. 

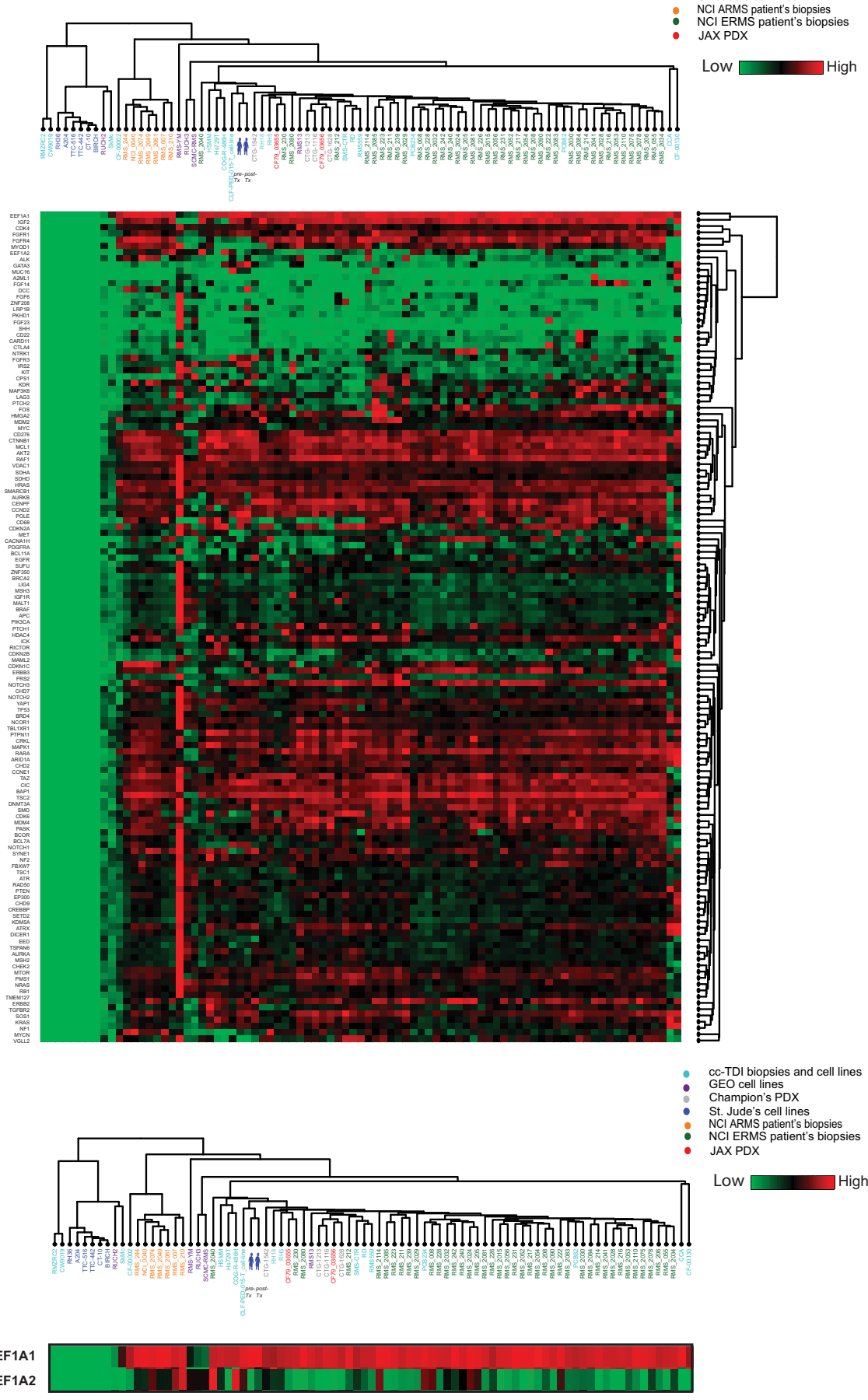

Figure 8. Unsupervised clustering of gene expression. Unsupervised clustering of embryonal rhabdomyosarcoma (ERMS) and non-rhabdomyosarcoma soft tissue sarcoma (NRSTS) samples with alveolar rhabdomyosarcoma (ARMS) samples harboring the PAX7:FOXO1 fusion gene as a control according to their expression of genes. Average-linkage clustering was performed on tumor samples from our CuReFAST registry (light blue), cell lines from Gene Expression Omnibus (GEO) (purple), cell lines from St. Jude Children's Research Hospital (blue), ARMS patient's biopsies from National Cancer Institute (orange), ERMS patient's biopsies from $\mathrm{NCl}$ (green), PDX mouse models from the Jackson Laboratory (red), and the patient's tumor sample (dark blue avatar) before treatment and after treatment. The matrix below the dendrogram has a sample in each column and gene in each row. The heat scale bar shows the relative gene expression per gene with red being the highest expression and green being the lowest. 
COLD SPRING HARBOR Molecular Case Studies
Translation factor eEF1A1 overexpression in a CIC-rearranged sarcoma case

Table 11. RNA sequencing results of CLF-PED-015-T

\begin{tabular}{llc}
\hline & \multicolumn{2}{c}{ CLF-PED-015-T } \\
\cline { 2 - 3 } & TPM & TU/(NM + 0.01) \\
\hline eEF1A1 & 9,892 & 48 \\
eEF1A2 & 48 & 0.019 \\
RPL32 & 4,546 & 14 \\
VGF & 13,679 & 341,975 \\
AP1M2 & 1,608 & 40,200 \\
ETV4 & 1,064 & 5,320 \\
\hline
\end{tabular}

approved CuReFAST initiative. We used average-linkage clustering and the Euclidean distance metric in RStudio Version 3.6.1 to develop a dendrogram with a legend that includes demographic data and somatic mutations. Average-linkage clustering and the Euclidean distance metric were used to develop the vertical dendrogram.

\section{Western Blot Analysis}

Cells were extracted in RIPA lysis buffer (ThermoFisher), supplemented with complete protease inhibitor and phosphatase inhibitor cocktail (ThermoFisher). After incubation on ice for $1 \mathrm{~h}$, samples were centrifuged at $13,000 \mathrm{~g}$ for $20 \mathrm{~min}$ at $4^{\circ} \mathrm{C}$. Protein samples were separated in SDS-PAGE gel and then transferred onto a $0.2-\mathrm{mm}$ polyvinylidene fluoride or polyvinylidene difluoride (PVDF) membrane using wet transfer method at $90 \mathrm{~V}$ for $90 \mathrm{~min}$. Primary antibodies anti-EF1a (Millipore \#05-235) and anti-GAPDH (Cell Signaling Cat \#2118S) were used with 1:1000 and 1:10,000 dilutions, respectively. Secondary antibodies HRP goat anti-rabbit (Vector Cat\# PI-1000) and HRP horse anti-mouse (Vector Cat\# PI-2000) were both diluted at 10,000. Proteins were detected using IVIS Lumina Imaging System.

\section{ADDITIONAL INFORMATION}

\section{Data Deposition and Access}

Raw data was deposited to our CuReFAST database, Foundation Medicine, and Human Longevity Inc. and can be accessed upon request. Raw sequencing data from our biopsy samples and cell lines were deposited to Gene Expression Omnibus (GEO) and European Genome-phenome Archive (EGA) under accession numbers GSE138269 and EGAS00001003981. The RNA and DNA sequencing data from this patient's normal and tumor samples will be submitted separately to EGA by the laboratory of Dr. E. Alejandro SweetCordero (UCSF). Variants found from this case have been submitted to ClinVar (https://www .nlm.ncbi.nih.gov/clinvar/) under accession numbers SCV001146981-SCV001146999.

\section{Ethics Statement}

Genetic studies and publication of clinical details were Institutional Review Board (IRB)approved, and full written informed consent was obtained from all participants. The parents provided consent for the proband.

\section{Acknowledgments}

This work was supported through Building Blocks to a Cure (1:1 match) crowdfunding campaign on Consano (https://consano.org/projects/building-blocks-to-a-cure-11-match/). 
COLD SPRING HARBOR Molecular Case Studies
Translation factor eEF1A1 overexpression in a CIC-rearranged sarcoma case
Competing Interest Statement

The authors have declared no competing interest.

Received October 6, 2019; accepted in revised form December 9, 2019.
N.E.B. was supported by research funding in memory of Ava Sentell. We greatly appreciate the participation and kind tissue donation of the patient and we thank the family for facilitating helpful discussion and participation in the study. We are also tremendously grateful for all the patients who kindly donated their tumor tissue as part of our CuReFAST initiative. We acknowledge those that have contributed to our sample collection: Dr. Marielle E. Yohe from the National Cancer Institute for providing the embryonal rhabdomyosarcoma cell line, CCA; Dr. Jonathon A. Fletcher from Brigham and Women's Hospital for providing the embryonal rhabdomyosarcoma cell line, RMS559; the Jackson Laboratory for providing WES and RNA sequencing data from PDX models; and the Gene Expression Omnibus (GEO) for sharing WES and RNA sequencing data form cell lines. We thank Dr. Atiya Mansoor, Dr. Amit Sabnis, and Dr. E. Alejandro Sweet-Cordero for providing their time and expertise.

\section{Author Contributions}

C.K., C.A.R., and N.E.B. designed the study. C.K., C.A.R., N.E.B., K.A.C., and E.R.R. collected and analyzed the data. C.K., C.A.R., A.N.H., N.E.B., A.D.W., E.R.R., and S.L.S. wrote the manuscript. C.K., C.A.R., N.E.B., K.A.C., T.G., A.N.H., A.D.W., E.D., S.H.R., S.L.S., and E.R.R. reviewed the manuscript.

\section{REFERENCES}

Antonescu CR, Owosho AA, Zhang L, Chen S, Deniz K, Huryn JM, Kao YC, Huang SC, Singer S, Tap W, et al. 2017. Sarcomas with CIC-rearrangements are a distinct pathologic entity with aggressive outcome: a clinicopathologic and molecular study of 115 cases. Am J Surg Pathol 41: 941-949. doi:10.1097/PAS 0000000000000846

Blanch A, Robinson F, Watson IR, Cheng LS, Irwin MS. 2013. Eukaryotic translation elongation factor 1- 1 inhibits p53 and p73 dependent apoptosis and chemotherapy sensitivity. PLoS One 8: e66436. doi:10.1371/ journal.pone.0066436

Doyle LA. 2014. Sarcoma classification: an update based on the 2013 World Health Organization Classification of Tumors of Soft Tissue and Bone. Cancer 120: 1763-1774. doi:10.1002/cncr.28657

Ferrari A, Miceli R, Rey A, Oberlin O, Orbach D, Brennan B, Mariani L, Carli M, Bisogno G, Cecchetto G, et al. 2011. Non-metastatic unresected paediatric non-rhabdomyosarcoma soft tissue sarcomas: results of a pooled analysis from United States and European groups. Eur J Cancer 47: 724-731. doi:10.1016/j.ejca .2010.11.013

Hong AL, Tseng YY, Cowley GS, Jonas O, Cheah JH, Kynnap BD, Doshi MB, Oh C, Meyer SC, Church AJ, et al. 2016. Integrated genetic and pharmacologic interrogation of rare cancers. Nat Commun 7: 11987. doi:10 .1038/ncomms11987

Hung YP, Fletcher CD, Hornick JL. 2016. Evaluation of ETV4 and WT1 expression in CIC-rearranged sarcomas and histologic mimics. Mod Pathol 29: 1324-1334. doi:10.1038/modpathol.2016.140

Italiano A, Sung YS, Zhang L, Singer S, Maki RG, Coindre JM, Antonescu CR. 2012. High prevalence of CIC fusion with double-homeobox (DUX4) transcription factors in EWSR1-negative undifferentiated small blue round cell sarcomas. Genes Chromosomes Cancer 51: 207-218. doi:10.1002/gcc.20945

Kao YC, Sung YS, Chen CL, Zhang L, Dickson BC, Swanson D, Vaiyapuri S, Latif F, Alholle A, Huang SC, et al. 2017. ETV transcriptional upregulation is more reliable than RNA sequencing algorithms and FISH in diagnosing round cell sarcomas with $\mathrm{ClC}$ gene rearrangements. Genes Chromosomes Cancer 56: 501-510. doi:10.1002/gcc.22454

Kawamura-Saito M, Yamazaki Y, Kaneko K, Kawaguchi N, Kanda H, Mukai H, Gotoh T, Motoi T, Fukayama M, Aburatani $\mathrm{H}$, et al. 2006. Fusion between $\mathrm{CIC}$ and DUX4 up-regulates PEA3 family genes in Ewing-like sarcomas with $\mathrm{t}(4 ; 19)(\mathrm{q} 35 ; \mathrm{q} 13)$ translocation. Hum Mol Genet 15: 2125-2137. doi:10.1093/hmg/ddl136

Liu X, Chen L, Ge J, Yan C, Huang Z, Hu J, Wen C, Li M, Huang D, Qiu Y, et al. 2016. The ubiquitin-like protein FAT10 stabilizes eEF1A1 expression to promote tumor proliferation in a complex manner. Cancer Res 76: 4897-4907. doi:10.1158/0008-5472.CAN-15-3118

Losada A, Muñoz-Alonso MJ, García C, Sánchez-Murcia PA, Martinez-Leal JF, Dominguez JM, Lillo MP, Gago F, Galmarini CM. 2016. Translation elongation factor eEF1A2 is a novel anticancer target for the marine natural product plitidepsin. Sci Rep 6: 35100. doi:10.1038/srep35100

Panagopoulos I, Gorunova L, Bjerkehagen B, Heim S. 2014. "The "grep" command but not FusionMap, FusionFinder or ChimeraScan captures the CIC-DUX4 fusion gene from whole transcriptome sequencing 
COLD SPRING HAR B OR

Molecular Case Studies
Translation factor eEF1A1 overexpression in a CIC-rearranged sarcoma case data on a small round cell tumor with $\mathrm{t}(4 ; 19)(q 35 ; q 13)$. PLoS One 9: e99439. doi:10.1371/journal.pone .0099439

Spunt SL, Skapek SX, Coffin CM. 2008. Pediatric nonrhabdomyosarcoma soft tissue sarcomas. Oncologist 13: 668-678. doi:10.1634/theoncologist.2007-0182

Tawbi HA-H, Burgess MA, Crowley J, Van Tine BA, Hu J, Schuetze S, D'Angelo SP, Attia S, Priebat DA, Okuno $\mathrm{SH}$, et al. 2016. Safety and efficacy of PD-1 blockade using pembrolizumab in patients with advanced soft tissue (STS) and bone sarcomas (BS): results of SARC028-a multicenter phase II study. J Clin Oncol 34: 11006. doi:10.1200/JCO.2016.34.15_suppl.11006

Watson S, Perrin V, Guillemot D, Reynaud S, Coindre JM, Karanian M, Guinebretière JM, Freneaux P, Le Loarer F, Bouvet M, et al. 2018. Transcriptomic definition of molecular subgroups of small round cell sarcomas. J Pathol 245: 29-40. doi:10.1002/path.5053 


\section{COLD SPRING HARBOR Molecular Case Studies}

\section{Undifferentiated small round cell sarcoma in a young male: a case report}

Cora A. Ricker, Noah E. Berlow, Kenneth A. Crawford, et al.

Cold Spring Harb Mol Case Stud 2020, 6: a004812

Access the most recent version at doi: $10.1101 / \mathrm{mcs} . a 004812$
Supplementary http://molecularcasestudies.cshlp.org/content/suppl/2020/01/29/mcs.a004812.D Material C1

References This article cites 15 articles, 2 of which can be accessed free at: http://molecularcasestudies.cshlp.org/content/6/1/a004812.full.html\#ref-list-1

License This article is distributed under the terms of the Creative Commons Attribution-NonCommercial License, which permits reuse and redistribution, except for commercial purposes, provided that the original author and source are credited.

Email Alerting Receive free email alerts when new articles cite this article - sign up in the box at the Service top right corner of the article or click here. 